\title{
Ultra low cycle fatigue of welded steel joints under multiaxial loading
}

\author{
Albano de Castro e Sousa \& Alain Nussbaumer \\ Resilient Steel Structures Laboratory - RESSLAB \\ Ecole Polytechnique Federale de Lausanne
}

\begin{abstract}
Ultra low cycle fatigue (ULCF) is a phenomenon that can be summarily defined as the deterioration of material properties under high amplitude cyclic loading, under which failure is reached typically within ten to twenty cycles. This phenomenon is observed in extreme loading scenarios such as earthquakes or exceptional winds where large cyclic strains are expected. Particularly sensitive are structural components that are designed to absorb the loading's energy through plastic dissipation, like moment or shear links in eccentrically braced frames. The demands on such components can vary significantly throughout its duration due to their variable multi-directional nature and, as such, it is important to assess the resistance of structural joints under multiaxial states. This paper will present an experimental campaign done on a welded high strength steel (S770QL) tube to plate joint. This joint was submitted to high cyclic bending, pure torsional and combined proportional bending and torsional loadings. Weld profile and strain measurements at the weld toe were performed using digital image correlation (DIC). An analysis of more than sixty test results will be presented so as to provide an adequate resistance model for ULCF life estimations.
\end{abstract}

\section{INTRODUCTION}

Extreme loading conditions such as earthquakes, exceptional wind dusts or tides are characterized by very high amplitude and multidirectional cyclic loads applied to a structure. Ultralow cycle fatigue (ULCF) arises in such cases and can be summarily defined as the deterioration of material properties due to repetitive loading at great amplitudes. In the case of welded steel joints, failure is reached typically in just a few number of cycles - less than ten or twenty (Kanvinde \& Deierlein 2004). Examples of structures susceptible to this problem include eccentrically braced frames, bridge piers, liquid storage tanks (Tappauf \& Taras 2015),(Prinz \& Nussbaumer 2012), wind energy masts and pressure vessels, the failure of which can cause human, economic and environmental losses.

The most widely used ULCF models in steel design stem from physics based monotonic fracture models (Kanvinde \& Deierlein 2004). Despite the wide range of fracture criteria, the most ubiquitous is the void growth model (VGM) and it's sibling, the stress modified critical strain (SMCS). The VGM is a micromechanical model that is based on the fact that steel at the microstructural level, due to the presence of inclusions and second phase particles, can develop with loading voids that grow and coalesce resulting in the formation of small internal cracks, precipitating frac- ture.

ULCF appears in this context as a way of estimating the fracture plastic strain or critical damage index, given that the material has been subjected to cyclic loading. In (Kanvinde \& Deierlein 2004) it is proposed to consider in the monotonic fracture criterion a factor that reflects the damage process that occurs during loading. This led to the adaptation of the VGM to form a cyclic void growth model (CVGM).

Issues with the applicability of the CVGM for multiaxial states notwithstanding, the assumption that failure is governed by the crack initiation process is critically important to the application of micromechanically based models in the assessment of structural components.

The component level tests presented herein consist of welded high strength (S770QL) tube to plate specimens subjected to a variety of loading conditions. These conditions were chosen so as to provide a wide set of directional loadings under both constant and variable amplitude.

Chiefly important in the resistance characterization of these tests is the use of digital image correlation (DIC). Very localized strain measurements were taken at the surface of the weld toe, the results of which are of great importance in framing an appropriate failure criterion. It was found that using measurements within cycles with no visible surface cracks, an extrapolation to total life estimations is possible using 


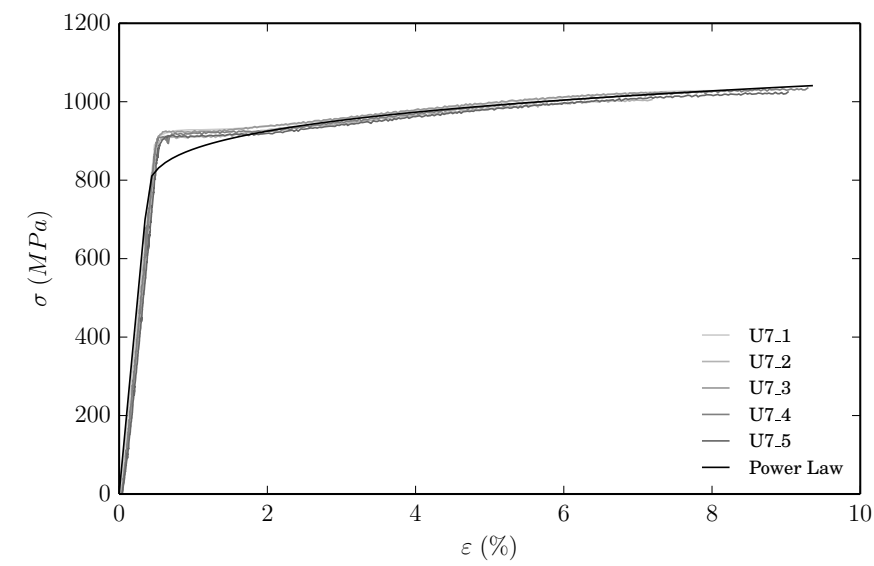

Figure 1: True Stress vs True Strain Curve for Uniaxial Tests and Fitted Power Law

the Manson-Coffin criterion with an acceptable level of scatter for design purposes.

\section{METHODOLOGY}

\subsection{Material properties}

The material studied in this paper is a high strength structural steel S770QL. Basic properties were determined using well established methods. Small specimens were cut out of the same circular hot rolled seamless tubes used in the component level tests.

It's chemical composition is presented in Table 1 , as provided by the inspection certificate, and later confirmed by Energy Dispersed X-ray Spectroscopy $(\mathrm{EDX})$ conducted at the Interdisciplinary Center for Electron Microscopy at EPFL (CIME). It is a quenched and tempered steel with martensitic microstructure and a grain size between 5 to $15 \mu \mathrm{m}$. Elasto-plastic mechanical properties were determined using smooth round bar tensile tests of $6 \mathrm{~mm}$ diameter in accordance with (ASTM 2013). 5 tests were conducted and the average results are summarized in Table 2. Here, $\sigma_{y}$ is the yield stress, $\sigma_{u}$ is the ultimate stress, $\mathrm{E}$ the modulus of elasticity, $\Delta \mathrm{L} / \mathrm{L}$ the elongation, and $\mathrm{K}$ and $\mathrm{n}$ the coefficients of a power hardening law defined in Eq.1,

$\sigma= \begin{cases}E \varepsilon^{e} & : \varepsilon \leq \varepsilon^{e} \\ \sigma_{y}+K\left(\varepsilon^{p}\right)^{n} & : \varepsilon>\varepsilon^{e}\end{cases}$

Figure 1 shows the results of the 5 tests and an average power law (considering a yield stress of $700 \mathrm{MPa}$ ) in true stress/true strain. These measures were taken as Eq. 2,3 from the engineering stress and strain measured in the tests up to necking of the specimens.

$\sigma=\sigma_{e n g}\left(1+\varepsilon_{e n g}\right)$

$\varepsilon=\ln \left(1+\varepsilon_{\text {eng }}\right)$

\subsection{Component level experiments}

A welded tube to plate joint was chosen to study ULCF behavior under multiaxial states under constant and variable amplitude loading. The primary reason to use this kind of specimens is the uniformity with which one can apply a shear stress/strain field in the area of interest, namely the weld toe, thus avoiding issues that can arise from stress/strain risers (primarily corners) in the load transfer process in planar plate specimens.

Figures 2 and 3 present the test setup (all dimensions are in millimeters). The test setup consists of a welded tube $(101.6 \times 10 \mathrm{~mm})$ to plate specimen bolted to a stiffened HEB360 column. The specimen is loaded in bending and torsion with the use of two hydraulic actuators. These actuators have $+/-200 \mathrm{kN}$ nominal capacity and $+/-250 \mathrm{~mm}$ stroke. The first actuator, used for bending, has a jack head with a circular opening of $105.0 \mathrm{~mm}$ diameter through which the specimen can pass. This opening sits on a spherical mount that is able to rotate on all three-axes. Torsion is applied by a second actuator, residing in a plane perpendicular to the tube's longitudinal axis, through a lever arm. The nominal length between axes is 440 $\mathrm{mm}$. This arm was custom built with four massive blocks of steel carved with a circular concave shape of diameter equal to the tube's own. These pieces constitute two pairs of clamps that are then prestressed together so as to be able to mobilize a significant amount friction between the surfaces of the blocks and the tube. Each pair reside on opposite sides of the bending jack's head. The blocks are then welded to two 'I' beams and subsequently connected to the torsion actuator.

In order to concentrate yielding near the weld region, the section was reduced from $10 \mathrm{~mm}$ to $8 \mathrm{~mm}$ according to the geometry outlined in Fig.4.

With respect to instrumentation, two vertical and one horizontal inductive displacement sensors were used to quantify slipping of the base plate in relation to the column. Two inclinometers were also used: one attached to the base plate and another connected to a section of the tube at a specific length. Digital image correlation was used to measure both the geometry and surface strain history of the weld toe region. This system is made of two Manta G-504B/C cameras with a $2452 \times 2056$ pixel CCD sensor used in tandem with two Fujinon $50 \mathrm{~mm}$ focal length lenses. Images were processed using the software Video Image Correlation in 3-D (VIC3D v7.0) (McGowan, Ambur, Hanna, \& McNeill 2001). The position of the cameras with respect to the weld toe and the size of the camera sensor set the resolution of the system, which in this setup makes 1 pixel correspond to approximately $0.03 \mathrm{~mm}$. Key options step size and filter size were set to 7 pixels and 7 data points, respectively, across all specimens so as to provide a uniform basis of comparison. This essentially means that full-field calcu- 
Table 1: Chemical Composition of S770QL

\begin{tabular}{cccccccccccc}
\hline $\mathrm{C}$ & $\mathrm{Mn}$ & $\mathrm{Si}$ & $\mathrm{Ni}$ & $\mathrm{Cr}$ & $\mathrm{Mo}$ & $\mathrm{V}$ & $\mathrm{Cu}$ & $\mathrm{P}$ & $\mathrm{S}$ & $C_{e q}^{I I W}$ & $C_{e q}^{A W S}$ \\
\hline $0.16 \%$ & $1.44 \%$ & $0.39 \%$ & $0.54 \%$ & $0.10 \%$ & $0.37 \%$ & $0.07 \%$ & $0.17 \%$ & $0.012 \%$ & $0.002 \%$ & $0.55 \%$ & $0.61 \%$ \\
\hline
\end{tabular}

Table 2: Summary table for smooth specimens

\begin{tabular}{rccccc}
\hline & $\sigma_{y}(\mathrm{MPa})$ & $\sigma_{u}(\mathrm{MPa})$ & $\mathrm{E}(\mathrm{GPa})$ & $\Delta \mathrm{L} / \mathrm{L}$ & $\mathrm{K}(\mathrm{MPa})$ \\
\hline Mean & 951 & 1024 & 197 & $20.79 \%$ & 615 \\
StD & 7.89 & 11.02 & 4.0 & $1.35 \%$ & 0.245 \\
COV & $0.83 \%$ & $1.08 \%$ & $2.02 \%$ & $6.47 \%$ & 27.82 \\
\hline
\end{tabular}

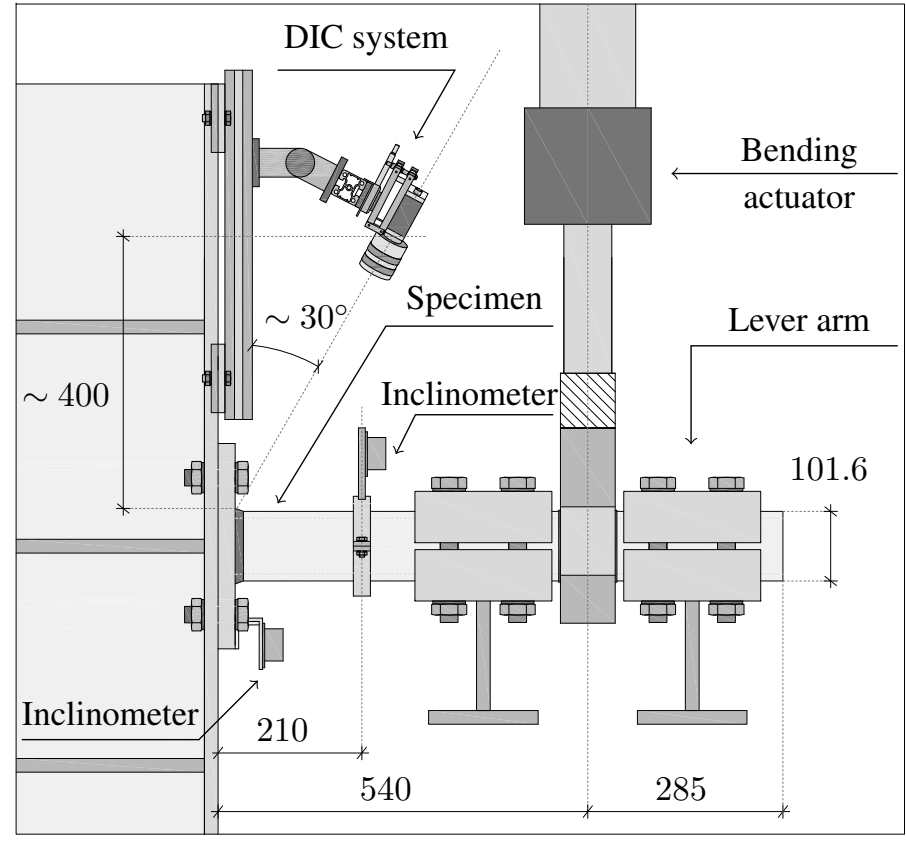

Figure 2: Tube to plate test setup - side view

lations are based on a grid of points spaced out of 7 pixels and then averaged over a window of 7 by 7 data points i.e. 49 by 49 pixels, i.e. approximately 1.5 by $1.5 \mathrm{~mm}$. Correlation of data points between images in the reference and the deformed configuration is more dependent on the speckle pattern on the surface of the tube, and thus subset size varies slightly between specimens.

Let us define $\delta$ as the displacement of the bending actuator minus the average displacement of the two vertical displacement sensors and the $3.4 \mathrm{~mm}$ maximum clearance in the spherical mount, and $\theta$ as the difference between the angles measured in the two inclinometers. Tests were conducted under displacement and rotation control: the controlling variable for the bending actuator was its head's displacement (not $\delta$ ); the displacement of the torsion actuator was controlled by $\theta$.

Table 3 presents the test matrix with 40 tests in constant amplitude (CA) proportional loading. Five different ratios between $\delta$ and $\theta$ were used and can be seen graphically in Fig. 5. Variable amplitude (VA) tests were also conducted under two types load histories: VA1 alternates between CA1 and CA2 and VA2 performs block loading of 3 cycles between CA1 and CA2. 3 specimens under both VA1 and VA2 for load

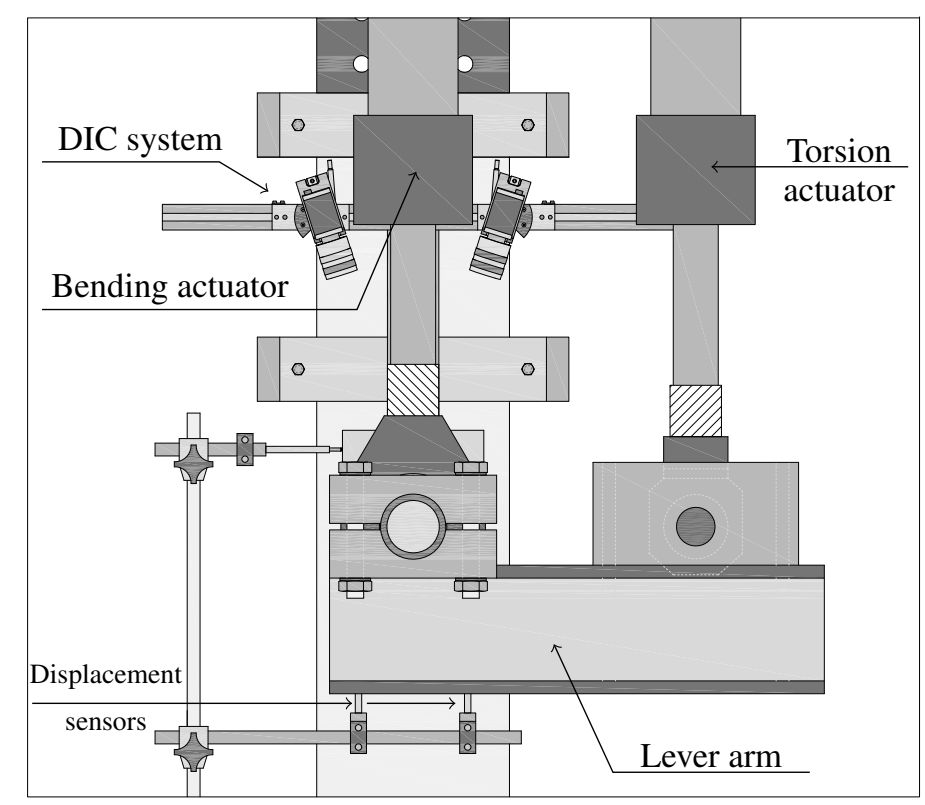

Figure 3: Tube to plate test setup - front view

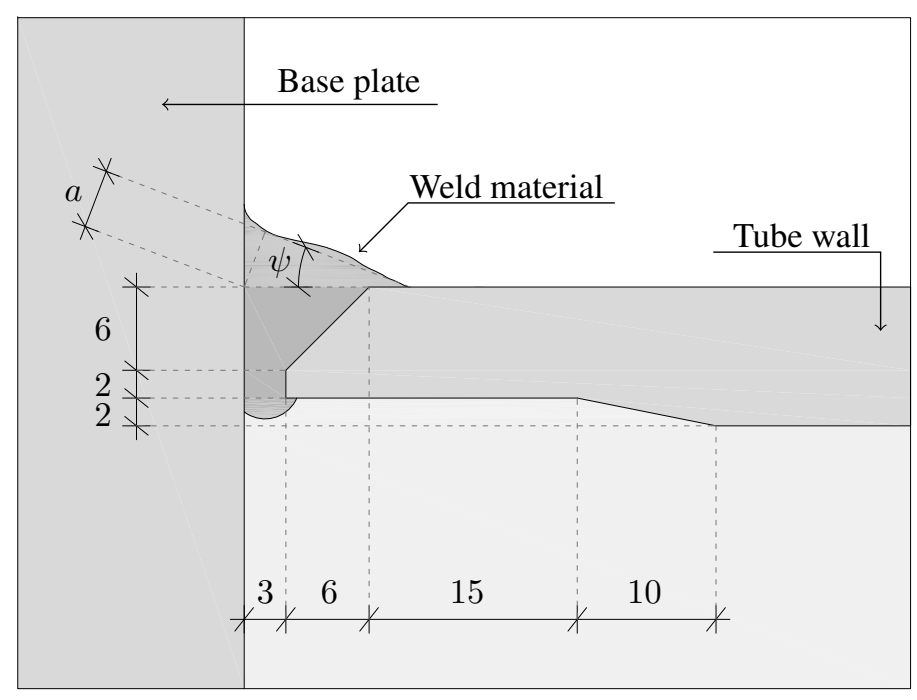

Figure 4: Detail of weld region 
Table 3: Constant amplitude test matrix

\begin{tabular}{ccccc}
\hline Type & Amp. & $\theta($ Deg $)$ & $\delta(\mathrm{mm})$ & Qty \\
\hline A (Torsion) & CA1 & 5.50 & 0.00 & 5 \\
A (Torsion) & CA2 & 4.10 & 0.00 & 4 \\
A (Torsion) & CA3 & 8.15 & 0.00 & 1 \\
A (Torsion) & CA4 & 2.20 & 0.00 & 1 \\
B (Bending) & CA1 & 0.00 & 38.50 & 4 \\
B (Bending) & CA2 & 0.00 & 33.50 & 4 \\
C & CA1 & 4.10 & 36.00 & 4 \\
C & CA2 & 3.50 & 31.00 & 4 \\
D & CA1 & 5.60 & 32.00 & 3 \\
D & CA2 & 4.00 & 23.00 & 4 \\
D & CA3 & 4.55 & 26.25 & 1 \\
F & CA1 & 4.70 & 14.00 & 2 \\
F & CA2 & 3.40 & 10.00 & 3 \\
\hline & & & Total & 40
\end{tabular}

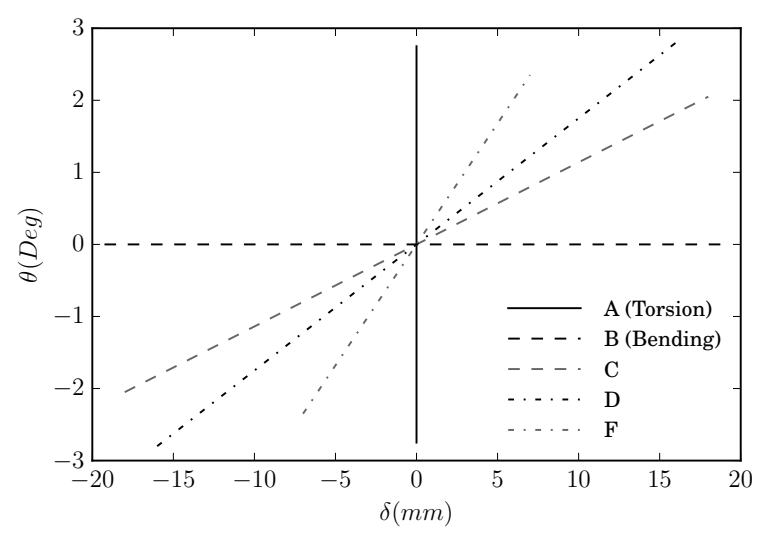

Figure 5: Constant amplitude load paths

ratios $\mathrm{A}, \mathrm{B}, \mathrm{C}$ and $\mathrm{D}$ were performed, totaling $24 \mathrm{VA}$ tests. The grand total of CA and VA is 64 tests.

Crucially important in the interpretation of results is the definition of what constitutes the failure of the specimen. It was found that in the majority of cases the detection of a visible crack (from the camera pictures) at the same time did not correspond to a significant drop in the load being applied by the actuators, i.e. a significant drop in resistance of the component. This led to the adoption of the following failure criterion: failure occurs when there is a $10 \%$ drop in the resistance, as measured by the force applied by the actuators.

\subsection{Finite element simulations}

Finite Element (FE) simulations of the tube to plate tests was carried out using Abaqus 6.11-2 and a nonlinear kinematic material model. Details of the implementation of the plasticity model are out of the scope of this paper but a short summary follows. The yield function, $\phi$, taking into account kinematic hardening, is expressed by Eq. 4.

$$
\phi=\sqrt{\frac{3}{2}\left(\bar{\sigma}^{\prime}-\bar{\alpha}^{\prime}\right):\left(\bar{\sigma}^{\prime}-\bar{\alpha}^{\prime}\right)}-\sigma_{y}
$$

$\sigma_{v m}=\sqrt{\frac{3}{2} \bar{\sigma}^{\prime}: \bar{\sigma}^{\prime}}$

where $\sigma_{v m}$ is the Von Mises stress at a material point. The terms under the square root in $\phi$ is very similar to $\sigma_{v m}$ except that the Frobenius sum (semicolon) is done on the deviatoric stress tensor, $\bar{\sigma}^{\prime}, \mathrm{mi}^{-}$ nus the total deviatoric back stress tensor, $\bar{\alpha}=\sum \bar{\alpha}_{k}$, with $\mathrm{k}$ being the $\mathrm{k}$-th backstress.

As plastic strain develops, hardening takes place. Hardening is often expressed as a function of a single scalar variable, the equivalent plastic strain, defined in rate terms in Eq. 6.

$\dot{\varepsilon}_{e q}^{p}=\sqrt{\frac{2}{3} \dot{\varepsilon}^{p}: \dot{\bar{\varepsilon}}^{p}}$

The k-th backstress evolution law is given by the Eq.7,

$\dot{\bar{\alpha}}_{k}=C_{k} \dot{\bar{\varepsilon}}^{p}-\gamma_{k} \bar{\alpha}_{k} \dot{\varepsilon}_{e q}^{p}$

where $C_{k}$ and $\gamma_{k}$ are material parameters representing a linear and a relaxation component of the backstress respectively. Indeed, the relaxation term in effect limits the values of the backstress to a certain value for sufficiently high equivalent plastic strain. All the examples that follow use parameters fitted to the power law represented in Fig.1 extrapolated to higher strains.

\section{RESULTS}

\subsection{Component level experiments}

One key result of this experimental program is the fact that surface cracks were detected much before failure of the component occurs. It is useful to acknowledge two factors in what constitutes the detection of a surface in these tests. The first is that this was done exclusively with pictures taking by the DIC system, which means that cracks might have developed elsewhere along the periphery of the weld toe. The second is that there is a limitation on the perception of what is a crack. At the scale that the pictures were taken, this limit can arguably be around $0.1 \mathrm{~mm}$ of surface opening. Also noteworthy is the fact that for pure torsion tests it is much harder to detect a surface crack due to both limited surface opening and higher probability of a critical crack happening elsewhere. As such, one can reasonably conclude that what follows constitutes an upper bound for an initiation criterion: the initiation ratio is the ratio between the number of cycles that a crack is detected and the number of cycles to failure $\left(N_{f}\right)$.

Considering Fig. 6, one can reasonably conclude that the initiation process constitutes, in rough terms, only half to a third of the total life.

Although equivalent strain models are not considered to be generally applicable (Socie \& Marquis 


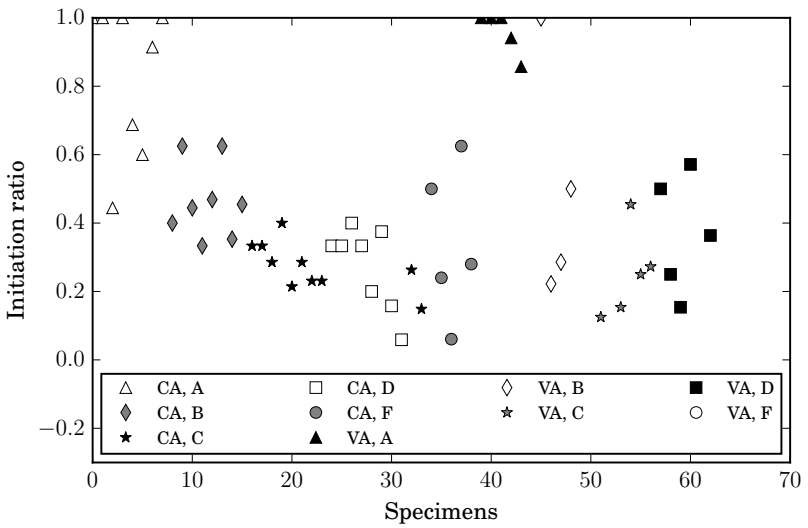

Figure 6: Initiation ratios with respect to total life by test type

1999), good agreement was found in expressing test results in these terms. Let us define equivalent strain as,

$\varepsilon_{e q}=\operatorname{sign}\left(\varepsilon_{i j}\right) \sqrt{\frac{2}{3} \bar{\varepsilon}: \bar{\varepsilon}}$

Note the differences between Eq.8 and Eq. 6 . Firstly Eq. 8 is expressed in terms of the total strain tensor $\left(\bar{\varepsilon}=\bar{\varepsilon}^{e}+\bar{\varepsilon}^{p}\right)$, which presupposes that the elastic strain tensor is small when compared with the plastic strain. Secondly it expresses a base quantity as opposed to a rate quantity. Lastly, it is endowed with the sign of the most relevant strain tensor quantity with respect to the loading e.g. for a pure torsion loading occurring in plane xy $\varepsilon_{i j} \equiv \varepsilon_{x y}$. The sole reason for this is to allow to keep track of the direction of the strain tensor with respect to the loading.

With the equivalent strain defined in such a way, it is possible to define a time history from DIC surface measurements in the following way: keeping in mind the assumption that elastic strains are small with respect to the plastic, one can evoke the condition of volume conservation which states that $\operatorname{trace}\left(\varepsilon^{p}\right)=0$ to approximate the strain depth component; secondly since there is no yielding in shear components except the one in the circumferential direction these are taken to be zero. Once a time history of this quantity is established one can count the number of cycles much in the same way as one does with a rainflow algorithm in high-cycle fatigue.

The fact that very local measurements are used instead of nominal section estimations, gives $\varepsilon_{e q}$ added significance even if for no other reason than the added resolution in capturing the material behavior. For instance, consider the same CA loading. A nominal strain approach would give the same $\Delta \varepsilon_{e q}$. However, when taking local measurements one is also taking into account local geometric and material properties that can affect significantly $\Delta \varepsilon_{e q}$.

The physical rationalization behind this quantity is that for a perfectly plastic material, the dissipation between the trough and peak of a cycle is the same using this quantity with the Von Mises stress or using the stress and strain tensors.

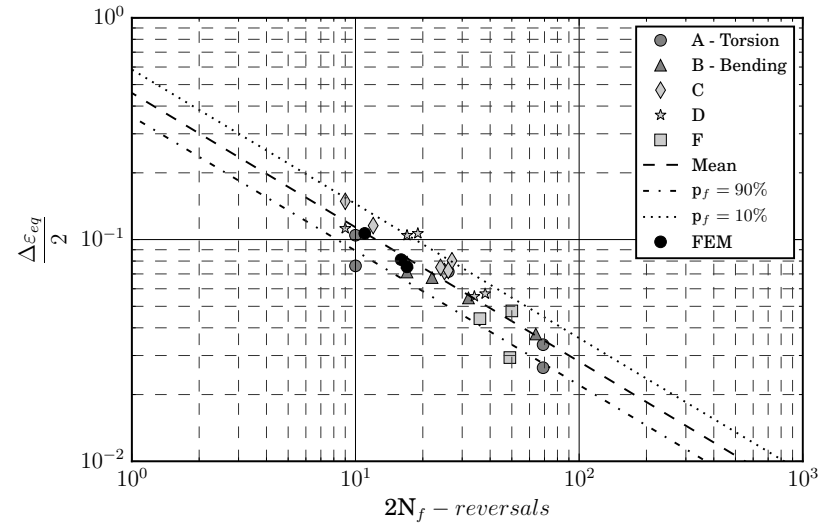

Figure 7: Manson-Coffin diagram in equivalent strain amplitude

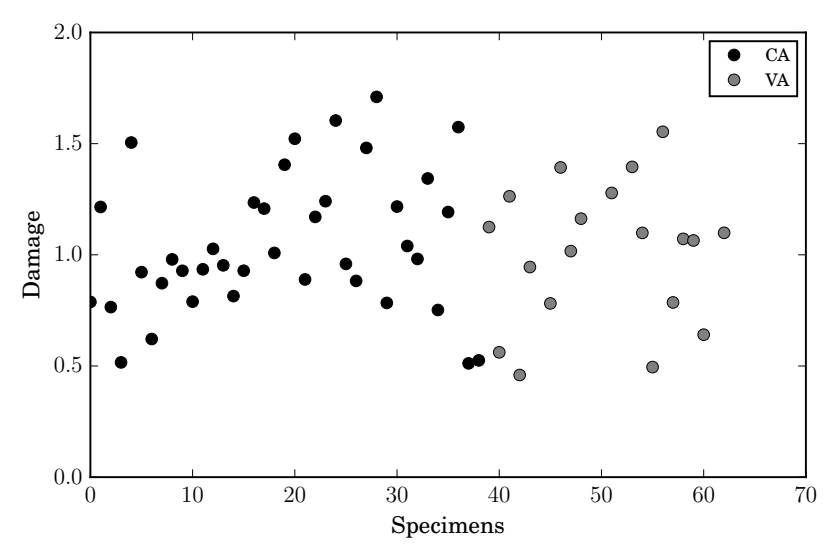

Figure 8: Damage given by Miner's rule per specimen

Let us consider the Manson-Coffin type relation in Eq.9.

$\frac{\Delta \varepsilon_{e q}}{2}=b\left(2 N_{f}\right)^{c}$

where, $b$ is the fatigue ductility coefficient and $c$ the ductility exponent (between -0.5 and -0.7 in many metals (ASM International 1996) ).

Fig. 7 shows the results of constant amplitude tests according to the assumptions previously stated. The ductility $c$ was found to be $\simeq-0.6$, well within expected bounds.

An important detail to mention is that the equivalent strain range can only be established by DIC within the initiation regime defined earlier for two main reasons: the first, once a surface crack starts to form these strains no longer meaningful; the second with successive cycles paint might peel off. Therefore, the $\Delta \varepsilon_{e q}$ in Fig. 7 is obtained only within early cycles and then assumed to hold its relevancy in the estimation of total life according to Eq.9.

With a good basis for CA tests established, the problem of VA can be addressed in the form of a damage sum according to the Palmgren-Miner rule. Fig. 8 shows the damage accumulation for both CA and VA loadings. The average value of damage is $\simeq 1.0$ with a coefficient of variation $(\mathrm{CoV})$ of $\simeq 30 \%$. These are fairly acceptable results in fatigue life terms. 


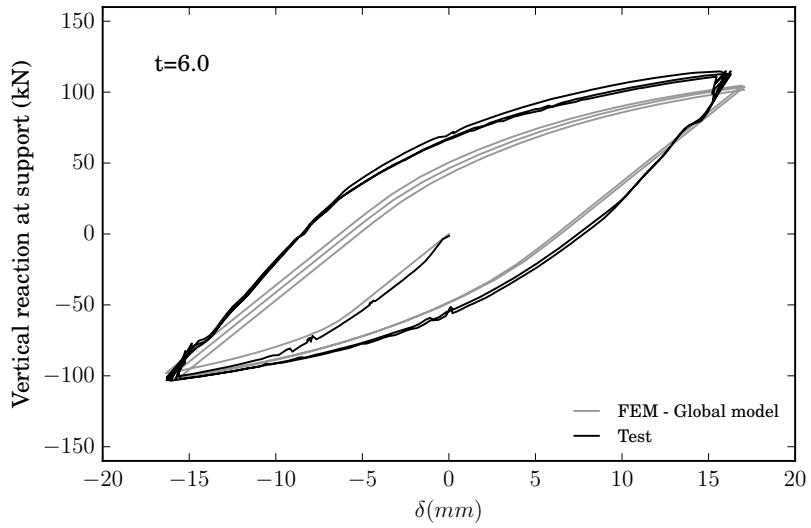

Figure 9: Hysteresis curve for specimen D7CA1_2 at load step 6 (force - displacement)

\subsection{Comparison with finite element simulations}

A successful estimation of strain histories with FE analyses requires two conditions: the first, an accurate simulation of the component level force-displacement hysteretic curve; the second, reproducing the strain history measured by DIC with sufficient accuracy.

Fig. 9 and 10 compare the forcedisplacement/torque-rotation curves measured for loading $\mathrm{C}$ at $\mathrm{CA} 1$ for specimen \#2. Considering that potentially relevant approximations were made (such as a simplification of the weld toe geometry and the assumption that the base material properties are representative of the material present at the weld), the hysteresis curve of the test and the FE simulation match quite well.

To capture small scale yielding, it was found that both element size and type are quite important. Indeed, it was necessary to use submodelling techniques in order to achieve sufficiently refined meshes. Curiously enough, the closest distance between element nodes shows a sign of tending to the DIC step size so that the strains at the weld toe are of similar orders of magnitude both in the local measurements and FE simulations. Fig. 11 shows the equivalent strain history taken at the maximum amplitude point in both the DIC and FE simulation. In this case amplitudes are as follows: $\Delta \bar{\varepsilon}_{e q}^{D I C}=28.6 \%$ and $\Delta \bar{\varepsilon}_{e q}^{F E M}=$ 21.3\%. In Fig. 7 three simulations are plotted, each being a different load type (bending, torsion and 'D'). This shows a good agreement between the FEM estimations and the mean values of the Manson-Coffin model derived solely from DIC measurements.

\section{CONCLUSIONS}

In summary, the Manson-Coffin approach to lowcycle continues to be applicable in the ULCF regime. This approach in conjunction with an equivalent strain amplitude criterion provides a reasonable extension to fatigue life estimation in multiaxial states.

Sufficiently detailed finite element simulations seem to capture the local nature of the DIC measure-

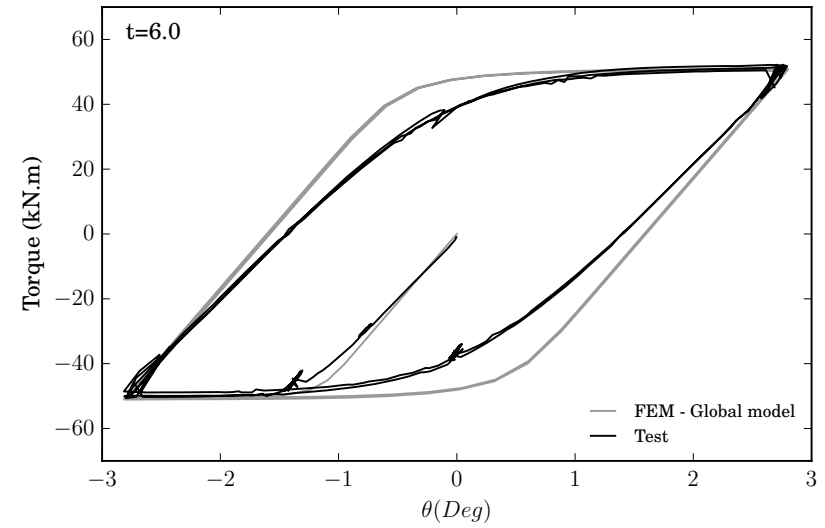

Figure 10: Hysteresis curve for specimen D7CA1_2 at load step 6 (torque - rotation)

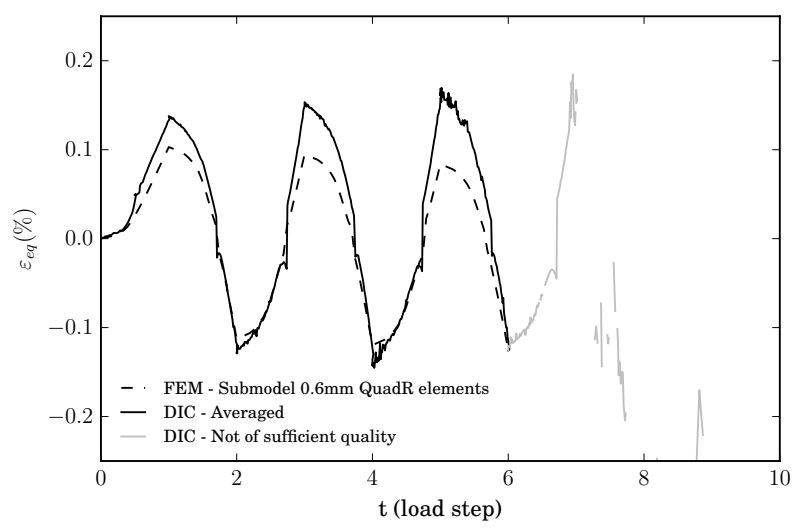

Figure 11: Equivalent strain history for specimen D7CA1_2

ments with enough accuracy to be considered as a suitable design approach.

Although this design framework is based on local strain measurements and therefore less dependent on specimen geometry, there is the risk it will suffer from the same component dependent deficiencies as other models because so much of the fatigue life is spent in propagation. Further analysis is needed to bound the application range of this approach.

\section{REFERENCES}

ASM International (1996). ASM Handbook Vol. 19 - Fatigue and Fracture.

ASTM (2013). E8/E8M - 13a - Standard Test Methods for Tension Testing of Metallic Materials 1.

Kanvinde, A. M. \& G. G. Deierlein (2004). Micromechanical Simulation of Earthquake Induced Fracture in Steel Structures. Technical Report 145, Blume Center, Stanford Univ., Stanford, CA.

McGowan, D. M., D. R. Ambur, T. G. Hanna, \& S. R. McNeill (2001). Evaluating the Compressive Response of Notched Composite Panels Using Full-Field Displacements. Journal of Aircraft 38(1), 122-129.

Prinz, G. S. \& A. Nussbaumer (2012, July). Fatigue analysis of liquid-storage tank shell-to-base connections under multiaxial loading. Engineering Structures 40, 75-82.

Socie, D. \& G. Marquis (1999). Multiaxial Fatigue. SAE International.

Tappauf, C. \& A. Taras (2015). Deformation and Strain Histories in Shell-to-Base Joints of Unanchored Steel Storage Tanks During Seismic Loading. In 8th International Conference on Behavior of Steel Structures in Seismic Areas, Shanghai. 